Sharif University of Technology
Scientia Iranica
SCIENTIA
I RAN I CA

\title{
Nonlinear free and forced vibrations of curved single-walled carbon nanotube on a Pasternak elastic foundation
}

\author{
M. Akbarzade*, A. Farshidianfar and M. Tahani \\ Department of Mechanical Engineering, Faculty of Engineering, Ferdowsi University of Mashhad, Mashhad, Iran.
}

Received 19 August 2015; received in revised form 28 August 2016; accepted 26 September 2016

\author{
KEYWORDS \\ Curved carbon \\ nanotube; \\ Nonlinear vibration; \\ Variational approach; \\ Hamiltonian \\ approach; \\ Perturbation theory.
}

\begin{abstract}
Based on elastic continuum mechanics, the nonlinear free and forced vibrations of an embedded single-walled carbon nanotube with waviness along its axis were analyzed. The single-walled carbon nanotube was embedded in a Pasternak elastic foundation. Two analytical approaches were utilized to obtain the frequency-amplitude relationship of the free vibrational model, and one other analytical approach was used to obtain the forced vibrations of the curved single-walled carbon nanotube on the Pasternak elastic foundation. Subsequently, a parametric study was performed to study the importance of different parameters, such as the amplitude of oscillation and the curvature radius, on the nonlinear behavior of the system. Also, a numerical simulation was carried out to obtain the results and investigate the accuracy of the analytical solution methods. Comparison of the results obtained by the proposed methods shows excellent agreement with those obtained by the numerical solution.

(C) 2016 Sharif University of Technology. All rights reserved.
\end{abstract}

\section{Introduction}

After the discovery of carbon nanotubes by Iigima [1], considerable attention has been devoted to carbon nanotubes, since they have the ability to revolutionize critical technologies owing to their remarkable mechanical, physical, and electrical properties [2].

Carbon nanotubes (CNTs) are perfect materials for a wide range of applications [3-5]. Carbon nanotubes possess excellent mechanical properties such as extremely high strength, stiffness, and resilience.

Deep understanding of the dynamic behavior of carbon nanotubes is essential for designing of novel nano devices. The natural frequencies of CNTs play an important role in nano mechanical resonators. It

\footnotetext{
*. Corresponding author. Tel.: +985138806055

E-mail addresses: mehdiakbarzade@yahoo.com ( $M$.

Akbarzade); farshid@um.ac.ir (A. Farshidianfar);

mtahani@um.ac.ir (M. Tahani)
}

is important to have accurate theoretical models for the natural frequencies and mode shapes of carbon nanotubes for several reasons. For example, if the nanotubes are to be used as nano mechanical resonators, the oscillation frequency is a key property of the resonator. Moreover, the effective elastic modulus of a nanotube may be indirectly determined from its measured natural frequencies or mode shapes if a sufficiently accurate theoretical model is used [6-9].

In modeling the dynamic behavior of carbon nanotubes, a number of investigators have adopted a continuum approach using classical beam assumptions and cylindrical shell theories to interpret experimental findings $[10,11]$. Continuum simulations require much less computational effort than the molecular dynamics simulations and predicting the behavior of nanostructures through continuum simulation is much cheaper than studying their behavior through experimental verification.

Recent theoretical and experimental studies show 
that the deformation of CNTs is nonlinear in nature. $\mathrm{Fu}$ et al. [12] investigated the nonlinear free vibration of embedded single- and multiple-walled CNTs. By using the incremental harmonic balanced method, they observed that as vibration amplitude increased, the nonlinear natural frequency increased for uniform simply supported single- and double-walled CNTs.

The nonlinear vibrations of embedded singlewalled carbon nanotube with geometrical imperfections, when subjected to a harmonic load, were investigated by Wang et al. [13] using the nonlocal Timoshenko beam theory. The effects of the nonlocal parameters, namely the elastic medium constants, waviness ratios, and the material lengths, on the dynamic response of their objective systems were analyzed.

The effects of a single-edge crack and its location on the buckling loads, natural frequencies, and the dynamic stability of circular curved beams were numerically investigated by Karaagac et al. [14] using the finite element method and the energy approach. Free vibrations of non-uniform thin curved arches and rings, using the Adomian modified decomposition method, were studied by Shahba et al. [14].

The chaotic behavior of a curved carbon nanotube under harmonic excitation was investigated by Mayoof and Hawwa [15]. The problem was formulated on the basis of the elastic continuum mechanics theory, where the carbon nanotube was modeled as a harmonically excited beam under a transverse force, in their work.

Figures 1 and 2 show that these tiny structures are not usually straight, but rather have a certain degree of curvature or waviness along the length of nanotubes. The curved morphology is due to processinduced waviness during manufacturing processes, in addition to mechanical properties such as low bending stiffness and large aspect ratio. The waviness of the carbon nanotube is modeled as a sinusoidal curvature with a small rise function [16].

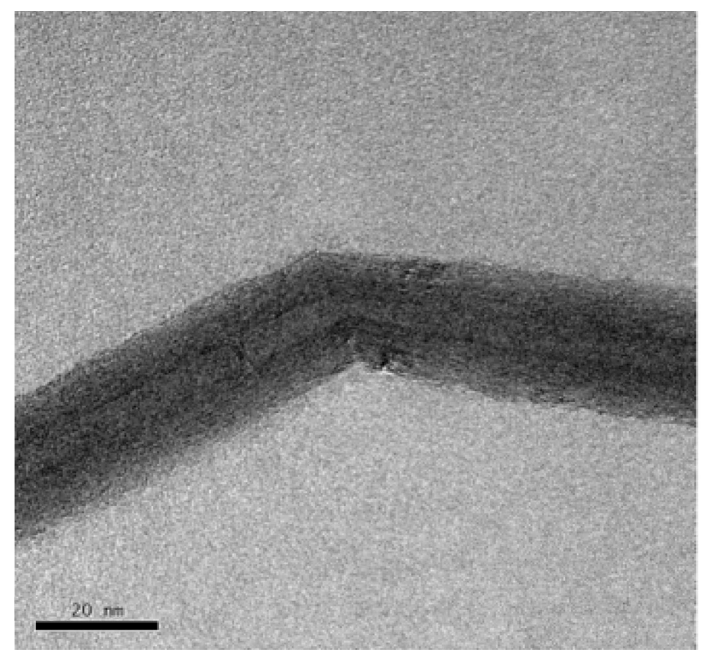

Figure 1. A curved carbon nanotube [16].

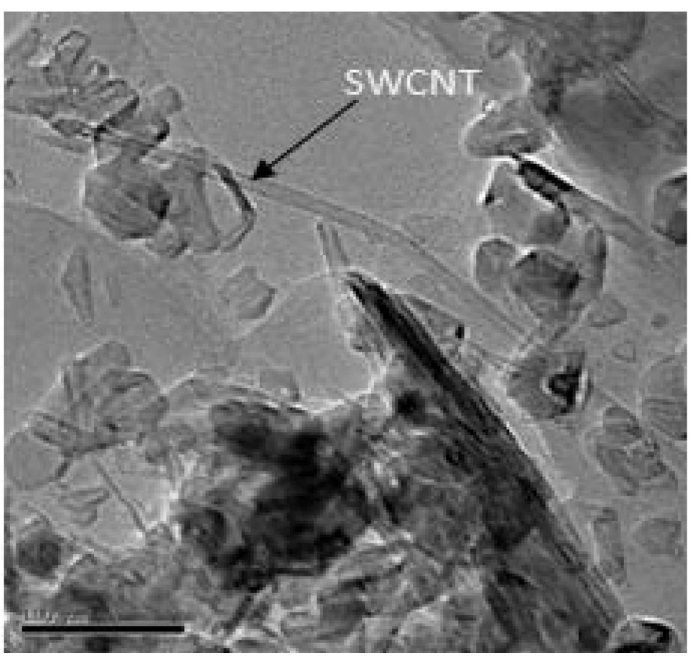

Figure 2. Transmission Electron Microscope (TEM) images of carbon nanotube, indicating the waviness [16].

In most of the previous studies, along with use of the classical beam theory for straight CNTs, resonance frequencies of CNTs have been measured experimentally. However, from photomicrographic images of CNTs, it is clear that they are not straight, and that they have some significant surface deviations, like waviness or the curvature associated with them.

In the present study, a nonlinear elastic beam model with midplane stretching nonlinearity is developed for the transverse vibration of a clampedclamped and curved Single-Walled CNT (SWCNT) on a Pasternak elastic foundation with a transverse harmonic excitation. We obtain the governing equations of motion for the harmonic transverse load of a curved SWCNT on a Pasternak elastic foundation; then, using a single-mode Galerkin approximation, we derive a second-order governing differential equation.

In this paper, calculating natural frequencies of curved single-walled carbon nanotube on a Pasternak elastic foundation and its relation with vibration amplitude are considered. Hamiltonian approach and coupled homotopy-variational formulation are employed to propose first-order approximate solutions for the governing equation of motion. For the first-order approximate solution, Hamiltonian approach can be applied with manual calculation without the requirement of advanced calculus.

Comparing the approximate frequency obtained by coupled homotopy-variational formulation with the numerical one, we see that this method is more accurate; but, it should be noted that in this method, the obtained residual contains three unknown parameters. In order to determine unknown parameters, we need three equations. Finding the unknown parameters in this method is more complex than finding the approximate frequency in Hamiltonian approach. Coupled homotopy-variational formulation is more accurate, 
but Hamiltonian approach can be applied with the requirement of easier calculus and is more suitable for the computer programming. We think these methods have great potential and can be applied to other types of nonlinear engineering problems.

In the present analysis, homotopy perturbation method is employed to seek the analytical solution to forced vibrations of curved single-walled carbon nanotube on a Pasternak elastic foundation. Homotopy perturbation method is an effective method to find a solution for a nonlinear differential equation. In this method, a nonlinear complex differential equation is transformed to a series of linear and nonlinear parts. These sets of equations are then solved iteratively. Finally, a linear series of the solutions completes the answer. We show that even the lowest order approximations obtained by the present theory are actually of high accuracy.

\section{Vibrational model}

A schematic diagram of a CNT embedded in a Pasternak elastic foundation is shown in Figure 3. The CNT is considered as a curved hollow cylindrical tube that has a sinusoidal curvature with a small rise function described by $Z=e \sin \left(\frac{\pi x}{L}\right)$ [15], where $e$ is the amplitude of its waviness. Assume that the transverse displacement is $w(x, t)$ in terms of the spatial coordinate, $x$, and the time variable, $t$. The kinetic energy of the carbon nanotube is given by:

$$
T=\frac{1}{2} \rho A \int_{0}^{L}\left(\frac{\partial w}{\partial t}\right)^{2} d x
$$

where $A$ is the cross sectional area, $\rho$ is the mass density, and $w$ is the transverse displacement. The potential energy is expressed as:

$$
\begin{aligned}
U= & \frac{1}{2} E A \int_{0}^{L}\left(\frac{\partial u}{\partial x}+\frac{1}{2}\left(\frac{\partial w}{\partial x}\right)^{2}+\frac{\partial Z}{\partial x} \cdot \frac{\partial w}{\partial x}\right)^{2} d x \\
& +\frac{1}{2} E I \int_{0}^{L}\left(\frac{\partial^{2} w}{\partial x^{2}}\right)^{2} d x+\frac{1}{2} \int_{0}^{L} k_{p}\left(\frac{\partial w}{\partial x}\right)^{2} d x \\
& +\frac{1}{2} \int_{0}^{L} k w^{2} d x
\end{aligned}
$$

where $E$ is the modulus of elasticity, $I$ is the area moment of inertia, $u$ is the axial displacement, $k_{p}$ is the shear foundation modulus, and $k$ is the elastic foundation modulus. Note that the first term in the potential energy equation is the energy due to the stretching of the beam, and other terms are due to the bending of the beam. Defining the Lagrangian $L$ as $L=$ $T-U$, applying the Hamilton's principle $\delta \int_{t_{1}}^{t_{2}} L d t=0$, integrating them to eliminate the longitudinal displace-

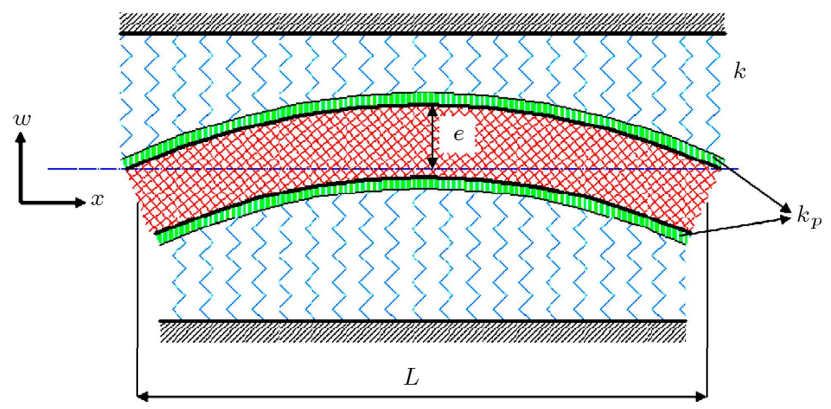

Figure 3. Model of an embedded curved carbon nanotube in an elastic Pasternak-type foundation.

ment, and imposing a harmonic transverse load lead to the following equation of motion [15]:

$$
\begin{aligned}
E I \frac{\partial^{4} w}{\partial x^{4}} & +k_{p} \frac{\partial^{2} w}{\partial x^{2}}+\rho A \frac{\partial^{2} w}{\partial t^{2}}+k w=F_{0} \cos \Omega_{0} t \\
& +\frac{1}{L} E A \int_{0}^{L}\left(\frac{1}{2}\left(\frac{\partial w}{\partial x}\right)^{2}+\frac{\partial Z}{\partial x} \cdot \frac{\partial w}{\partial x}\right)^{2} d x \\
& \cdot\left(\frac{\partial^{2} w}{\partial x^{2}}+\frac{\partial^{2} Z}{\partial x^{2}}\right)
\end{aligned}
$$

where $F$ is the spatial distribution of the transverse load and $\Omega_{0}$ is the frequency of applied transverse load [17].

Assuming that the CNT is clamped-clamped at the two ends, the boundary conditions are as follows:

$$
w(0, t)=\frac{\partial w(0, t)}{\partial x}=0, \quad w(L, t)=\frac{\partial w(L, t)}{\partial x}=0 .
$$

To perform an analysis of separation of variables, the transverse displacement can be written as:

$$
w(x, t)=W(x) T(t) .
$$

The basis function, $W(x)$, is assumed to take the fundamental mode shape of the linear vibration of the problem defined by Eqs. (3) and (4). Thus, according to the boundary conditions, the unknown function, $W(x)$, is given as:

$$
W(x)=\sqrt{\frac{2}{3}}\left[1-\cos \left(\frac{2 \pi x}{L}\right)\right] .
$$

Substituting this shape function, $W(x)$, and the curvature equation, $Z(x)$, into Eq. (3), multiplying the result by $W(x)$, and then integrating them over the domain $[0, L]$ lead to the following equation $[17]$ :

$$
\frac{d^{2} T(t)}{d t^{2}}+a T(t)+b T(t)^{2}+c T(t)^{3}=\sqrt{\frac{2}{3}} \frac{F_{0}}{\rho A} \cos \left(\Omega_{0} t\right)
$$

where: 


$$
\begin{aligned}
& a=\frac{4}{3} \frac{\pi^{2} k_{p}}{\rho A L^{2}}+\frac{128}{27} \frac{\pi^{2} E e^{2}}{\rho L^{4}}+\frac{k}{\rho A}+\frac{16}{3} \frac{\pi^{4} E I}{\rho A L^{4}}=\omega_{0}^{2} \\
& b=\frac{16 \sqrt{6}}{9} \frac{\pi^{3} E e}{\rho L^{4}}
\end{aligned}
$$

and:

$$
c=\frac{8}{9} \frac{\pi^{4} E}{\rho L^{4}} .
$$

The linear free vibrational frequency is assumed to be $\omega_{0}$ in Eq. (8), and $\omega_{0}^{2}=a$. The equation of motion can be normalized using the parameter $t=\frac{1}{\omega_{0}}$, the period of oscillation as a characteristic time, $r=d_{\text {out }}$, and the outer diameter of the carbon nanotube as a characteristic length. Hence, the non-dimensional form of the equation of motion becomes:

$$
\frac{d^{2} T(t)}{d t^{2}}+\alpha_{1} T(t)+\alpha_{2} T(t)^{2}+\alpha_{3} T(t)^{3}=F \cos (\Omega t),
$$

where:

$$
\begin{aligned}
& \Omega=\frac{\Omega_{0}}{\omega_{0}}, \quad \alpha_{1}=1 \quad \alpha_{2}=\frac{b r}{a}, \\
& \alpha_{3}=\frac{c r^{2}}{a}, \quad F=\sqrt{\frac{2}{3}} \frac{F_{0}}{r a \rho A L^{2}} .
\end{aligned}
$$

\section{Nonlinear free vibration analysis}

In the present analysis, two effective and convenient methods are employed to seek the analytical solution to nonlinear free vibration of curved single-walled carbon nanotube on a Pasternak elastic foundation.

\subsection{Application of the Hamiltonian approach}

This approach is a kind of energy method with a vast application in conservative oscillatory systems [18-24]. In order to clarify this approach, Eq. (11) can be rewritten in the following form:

$$
\frac{d^{2} T(t)}{d t^{2}}+\alpha_{1} T(t)+\alpha_{2} T(t)^{2}+\alpha_{3} T(t)^{3}=0
$$

According to Eq. (13), variational form can be easily obtained as follows [25]:

$$
\begin{aligned}
J= & \int_{0}^{\frac{2 \pi}{\omega}}\left(-\frac{1}{2}\left(\frac{d T(t)}{d t}\right)^{2}+\frac{1}{2} \alpha_{1} T(t)^{2}+\frac{1}{3} \alpha_{2} T(t)^{3}\right. \\
& \left.+\frac{1}{4} \alpha_{3} T(t)^{4}\right) d t
\end{aligned}
$$

Hamiltonian, therefore, can be written in the following form:

$$
\begin{aligned}
H= & \frac{1}{2}\left(\frac{d T(t)}{d t}\right)^{2}+\frac{1}{2} \alpha_{1} T(t)^{2}+\frac{1}{3} \alpha_{2} T(t)^{3} \\
& +\frac{1}{4} \alpha_{3} T(t)^{4}=\text { constant. }
\end{aligned}
$$

In Eq. (15), $\frac{1}{2}\left(\frac{d T(t)}{d t}\right)^{2}$ is the kinetic energy and $\frac{1}{2} \alpha_{1} T(t)^{2}+\frac{1}{3} \alpha_{2} T(t)^{3}+\frac{1}{4} \alpha_{3} T(t)^{4}$ is the potential energy. Integrating Eq. (15) with respect to $t$ from 0 to $T / 4$ yields:

$$
\begin{aligned}
\bar{H}= & \int_{0}^{\frac{2 \pi}{\omega}}\left(\frac{1}{2}\left(\frac{d T(t)}{d t}\right)^{2}+\frac{1}{2} \alpha_{1} T(t)^{2}+\frac{1}{3} \alpha_{2} T(t)^{3}\right. \\
& \left.+\frac{1}{4} \alpha_{3} T(t)^{4}\right) d t
\end{aligned}
$$

Oscillatory systems contain two important physical parameters, i.e., the frequency, $\omega$, and the amplitude of oscillation, $A$. Therefore, the following initial conditions are considered:

$$
T(0)=\left.A \quad \frac{d T(t)}{d t}\right|_{t=0}=0 .
$$

It is assumed that the initial approximate guess can be expressed as:

$$
T(t)=A \cos (\omega t) \text {. }
$$

Substituting Eq. (18) into Eq. (16) yields:

$$
\bar{H}=\frac{A^{2}}{\omega}\left(\frac{3}{64} A^{2} \pi \alpha_{3}+\frac{1}{8} \omega^{2} \pi+\frac{2}{9} A \alpha_{2}+\frac{1}{8} \pi \alpha_{1}\right),
$$

setting [25]:

$$
\frac{\partial}{\partial A}\left(\frac{\partial \bar{H}}{\partial(1 / \omega)}\right)=0
$$

The solution of the above equation yields an approximate frequency as a function of amplitude as:

$$
\omega_{H A}=\sqrt{\alpha_{1}+\frac{8}{3 \pi} A \alpha_{2}+\frac{3}{4} A^{2} \alpha_{3}} .
$$

\subsection{Application of the coupled method of homotopy perturbation method and variational formulation}

In this approach, combining the features of the homotopy concept with variational approach is proposed to find accurate analytical solutions for nonlinear oscillators [26-33]. In this method, according to the homotopy technique, a homotopy with an imbedding parameter, $p \in\left[\begin{array}{ll}0 & 1\end{array}\right]$, is constructed, as the zerothorder approximate solution is easy to be obtained. The second term is solved using the variational approach, where the frequency of the nonlinear oscillator can be obtained [27].

By considering the nonlinear oscillator, i.e. 
Eq. (13), the following homotopy can be constructed:

$$
\begin{aligned}
\frac{d^{2} T(t)}{d t^{2}} & +\omega^{2} T(t)+p\left(\left(\alpha_{1}-\omega^{2}\right) T(t)+\alpha_{2} T(t)^{2}\right. \\
& \left.+\alpha_{3} T(t)^{3}\right)=0
\end{aligned}
$$

when $p=0$, Eq. (22) becomes a linearized equation, $\frac{d^{2} T(t)}{d t^{2}}+\omega^{2} T(t)=0$, and when $p=1$, it turns out to be the original one. Assume that the periodic solution to Eq. (13) may be written as a power series in $p$ as [34]:

$$
T(t)=p^{0} T_{0}(t)+p^{1} T_{1}(t)+p^{2} T_{2}(t)+\ldots
$$

Substituting Eq. (23) into Eq. (22) and collecting terms of the same power of $p$ give:

$$
\begin{aligned}
\frac{d^{2} T_{0}(t)}{d t^{2}} & +\omega^{2} T_{0}(t)=0 \\
\frac{d^{2} T_{1}(t)}{d t^{2}} & +\omega^{2} T_{1}(t)+\alpha_{1} T_{0}(t)-\omega^{2} T_{0}(t)+\alpha_{2} T_{0}(t)^{2} \\
& +\alpha_{3} T_{0}(t)^{3}=0 .
\end{aligned}
$$

The solution of Eq. (24) is $T(t)=A \cos (\omega t)$, where $\omega$ will be identified from the variational formulation for $u$, which reads:

$$
\begin{aligned}
J= & \int_{0}^{\frac{2 \pi}{\omega}}\left(\alpha_{3} A^{3} \cos ^{3}(\omega t) T_{1}(t)+\alpha_{2} A^{2} \cos ^{2}(\omega t) T_{1}(t)\right. \\
& -\omega^{2} A \cos (\omega t) T_{1}(t)+\frac{1}{2} \omega^{2} T_{1}(t)^{2} \\
& \left.+\alpha_{1} A \cos (\omega t) T_{1}(t)-\frac{1}{2}\left(\frac{d T_{1}(t)}{d t}\right)^{2}\right) d t
\end{aligned}
$$

Next, the following trail is chosen:

$$
\begin{aligned}
T_{1}(t)= & B_{1}(\cos (\omega t)-\cos (5 \omega t))+B_{2}(\cos (2 \omega t) \\
& -\cos (6 \omega t)) .
\end{aligned}
$$

Substituting Eq. (27) into Eq. (26) leads to the result:

$$
\begin{aligned}
J\left(A, B_{1}, B_{2}, \omega\right)= & \frac{1}{\omega}\left(\frac{3}{4} A^{3} \pi B_{1} \alpha_{3}+\frac{1}{2} A^{2} \pi B_{2} \alpha_{2}\right. \\
& -A \omega^{2} \pi B_{1}-12 \omega^{2} \pi B_{1}^{2}-19 \omega^{2} \pi B_{2}^{2} \\
& \left.+A \pi B_{1} \alpha_{1}\right) .
\end{aligned}
$$

The stationary condition of Eq. (28) requires that [3537]:

$$
\frac{\partial J}{\partial B_{1}}=0, \quad \frac{\partial J}{\partial B_{2}}=0, \quad \frac{\partial J}{\partial \omega}=0 .
$$

Hence, the solutions of Eq. (29) are:

$$
B_{2}=\frac{1}{76} \frac{A^{2} \alpha_{2}}{\omega^{2}}
$$

and:

$$
B_{1}=\frac{1}{32} \frac{A^{3} \alpha_{3}}{\omega^{2}}-\frac{1}{24} A+\frac{1}{24} \frac{A \alpha_{1}}{\omega^{2}} .
$$

The first-order approximate solution is obtained as follows:

$$
\begin{aligned}
& \omega= \\
& \sqrt{-\frac{3}{4} A^{2} \alpha_{3}+\sqrt{\frac{9}{4} A^{4} \alpha_{3}^{2}+6 A^{2} \alpha_{1} \alpha_{3}+\frac{9}{19} A^{2} \alpha_{2}^{2}+4 \alpha_{1}^{2}-\alpha_{1}}}
\end{aligned}
$$

\section{Forced vibration}

The governing differential equation of curved singlewalled carbon nanotube on a Pasternak elastic foundation under harmonic excitation (see Figure 3 ) is:

$$
\frac{d^{2} T(t)}{d t^{2}}+\alpha_{1} T(t)+\alpha_{2} T(t)^{2}+\alpha_{3} T(t)^{3}=F \cos (\Omega t) \text {. }
$$

\subsection{Method of the solution}

In the present analysis, a very effective and convenient method (homotopy perturbation method [27]) is employed to seek the analytical solution of Eq. (33). Homotopy perturbation method can be applied to nonlinear vibrations and oscillations.

This method does not require linearization or a small parameter like the normal perturbation technique. The results will reveal the simplicity of the method. To illustrate the basic ideas of this method, we consider the following equation [27]:

$$
A(u)-f(r)=0, \quad r \in \Omega,
$$

with the boundary condition of:

$$
B\left(u, \frac{\partial u}{\partial n}\right)=0, \quad r \in \Gamma,
$$

where $A$ is a general differential operator, $B$ is a boundary operator, $f(r)$ is a known analytical function, and $\Gamma$ is the boundary of the domain $\Omega$. $A$ can be divided into two parts, which are $L$ and $N$, where $L$ is linear and $N$ is nonlinear. Eq. (34) can, therefore, be rewritten as follows:

$$
L(u)+N(u)-f(r)=0, \quad r \in \Omega .
$$

Homotopy perturbation is shown as follows:

$$
H(v, p)=(1-p)\left[L(v)-L\left(u_{0}\right)\right]+p[A(v)-f(r)]=0
$$

where:

$$
v(r, p): \Omega \times[0,1] \rightarrow R .
$$

In Eq. (37), $p \in[0,1]$ is an embedding parameter and $u_{0}$ is the first approximation that satisfies the boundary condition. We can assume that the solution of Eq. (37) can be written as a power series in $p$, as the following: 
Table 1. Comparison of Hamiltonian approach (Eq. (22)) and coupled method of homotopy-variational formulation (Eq. (23)) with the numerical solution $(A=1 \mathrm{~nm})$.

\begin{tabular}{ccccccccccc}
\hline & Case 1 & Case 2 & Case 3 & Case 4 & Case 5 & Case 6 & Case 7 & Case 8 & Case 9 & Case 10 \\
\hline $\boldsymbol{r}_{\text {in }}$ & 0 & 0.50 & 0.75 & 1.00 & 1.50 & 0 & 0.50 & 0.75 & 1.00 & 1.50 \\
$\boldsymbol{e}$ & 0.10 & 0.10 & 0.10 & 0.10 & 0.10 & 0.20 & 0.20 & 0.20 & 0.20 & 0.20 \\
$\boldsymbol{\alpha}_{\mathbf{2}}$ & 0.101 & 0.095 & 0.088 & 0.081 & 0.064 & 0.201 & 0.190 & 0.177 & 0.161 & 0.127 \\
$\boldsymbol{\alpha}_{\mathbf{3}}$ & 2.592 & 2.439 & 2.271 & 2.070 & 1.639 & 2.585 & 2.433 & 2.266 & 2.066 & 1.636 \\
$\boldsymbol{\omega}_{\mathrm{HA}}$ & 1.741 & 1.706 & 1.667 & 1.619 & 1.511 & 1.763 & 1.728 & 1.688 & 1.639 & 1.528 \\
$\boldsymbol{\omega}_{\mathrm{CM} \mathrm{HV}}$ & 1.716 & 1.682 & 1.644 & 1.598 & 1.493 & 1.715 & 1.681 & 1.643 & 1.597 & 1.492 \\
$\boldsymbol{\omega}_{\mathrm{Num}}$ & 1.715 & 1.682 & 1.642 & 1.595 & 1.492 & 1.713 & 1.680 & 1.641 & 1.595 & 1.491 \\
\hline
\end{tabular}

$$
v=v_{0}+p v_{1}+p^{2} v_{2}+\ldots
$$

and the best approximation for solution is:

$$
u=\lim _{p \rightarrow 1} v=v_{0}+v_{1}+v_{2}+\ldots
$$

In this study, Eq. (33) is rewritten as:

$$
\frac{d^{2}}{d t^{2}} T(t)+\alpha_{1} T(t)-F \cos (\Omega t)+p\left(\alpha_{2} T(t)^{2}+\alpha_{3} T(t)^{3}\right) .
$$

According to Eq. (39), the solution can be assumed as follows:

$$
T(t)=p^{0} T_{0}(t)+p^{1} T_{1}(t)+p^{2} T_{2}(t)+\ldots
$$

According to the HPM method, by substituting Eq. (42) into Eq. (41) and rearranging the subsequent result based on powers of $p$ terms, we can obtain:

$$
p^{0}:-F \cos (\Omega t)+\alpha_{1} T_{0}(t)+\frac{d^{2}}{d t^{2}} T_{0}(t)
$$

and:

$$
p^{1}: \alpha_{3} T_{0}(t)^{3}+\alpha_{2} T_{0}(t)^{2}+T_{1}(t) \alpha_{1}+\frac{d^{2}}{d t^{2}} T_{1}(t) .
$$

The following initial conditions are assumed:

$$
T_{0}(0)=\left.A \quad \frac{d T_{0}(t)}{d t}\right|_{t=0}=0
$$

By solving Eq. (43), we have the solution as:

$$
T_{0}(t)=\frac{\cos \left(\sqrt{\alpha_{1}} t\right)\left(A \omega^{2}-A \alpha_{1}+F\right)}{\omega^{2}-\alpha_{1}}-\frac{F \cos (\omega t)}{\omega^{2}-\alpha_{1}}
$$

For Eq. (44), the following initial conditions are assumed:

$$
T_{1}(0)=\left.0 \quad \frac{d T_{1}(t)}{d t}\right|_{t=0}=0
$$

By solving Eq. (44), we have the solution as shown in Box I.

The solution of the forced vibration in Eq. (33) is as follows:

$$
T(t)=\lim _{p \rightarrow 1} p^{0} T_{0}(t)+p^{1} T_{1}(t)+\ldots
$$

\section{Numerical results}

In order to assess the advantages and the accuracy of the proposed methods, we consider numerical RungeKutta method of order four to obtain the solution of the free and forced vibration problems. To this end, a computer program is developed, using MATLAB, to solve the equations numerically. The parameters of the material and geometry are taken as [17]:

$$
\begin{aligned}
& E=3.3 \mathrm{Tpa}, \quad \rho=2270 \mathrm{~kg} / \mathrm{m}^{3}, \\
& L=60 \mathrm{~nm}, \quad k_{p}=10^{-8} \mathrm{~N}, \\
& k=10^{7} \mathrm{~N}, \quad r_{\text {out }}=2 \mathrm{~nm} .
\end{aligned}
$$

In Table 1, the results obtained by Hamiltonian Approach (HA), Coupled Method of HomotopyVariational formulation (CMHV), and numerical solution for free vibration analysis of ten different case studies are compared.

It has been concluded that both results obtained by both methods are accurate and close to each other. In the coupled method of homotopy-variational formulation, the first-order approximates can be readily obtained with high accuracy. It is obvious that the variational approach provides a freedom of choice of trial function and gives us more information on the relation between frequency and amplitude.

These methods might prove versatile in engineering due to their simple solution procedures and high accuracy of results. We believe these methods have great potential and can be applied to other types of nonlinear problems.

The time history responses of the system, and also the comparison between hamiltonian approach and coupled method of homotopy-variational formulation for free vibration analysis in conjunction with numerical solution, are presented in Figures 4-7 for different parameters. By comparing the approximate response obtained by coupled homotopy-variational formulation with the numerical one, we see that coupled homotopyvariational formulation method is more accurate.

The influences of the curvature radius and ampli- 


$$
\begin{aligned}
& T_{1}(t)=\frac{1}{12} \frac{\cos \left(\sqrt{\alpha_{1}} t\right) F^{2}\left(4 \omega^{2} \alpha_{2}+3 F \alpha_{3}-4 \alpha_{1} \alpha_{2}\right)}{\alpha_{1}\left(\omega^{6}-3 \omega^{4} \alpha_{1}+3 \omega^{2} \alpha_{1}^{2}-\alpha_{1}^{3}\right)}-\frac{9}{32 \alpha_{1}^{(2 / 3)}\left(\Omega^{2}-\alpha_{1}\right)^{3}} F^{2}\left(-\frac{16}{27} \alpha_{2}\left(\sqrt{\alpha_{1}} \Omega^{2}-\alpha_{1}^{(3 / 2)}\right) \cos \left(2 \sqrt{\alpha_{1}} t\right)\right. \\
& \left.-\frac{1}{9} \alpha_{3} F \cos \left(3 \sqrt{\alpha_{1}} t\right) \sqrt{\alpha_{1}}+\alpha_{3} F \cos \left(\sqrt{\alpha_{1}} t\right) \sqrt{\alpha_{1}}+\frac{4}{3} F t \alpha_{3} \sin \left(\sqrt{\alpha_{1}} t\right) \alpha_{1}+\frac{16}{9} \alpha_{2}\left(\sqrt{\alpha_{1}} \Omega^{2}-\alpha_{1}^{(3 / 2)}\right)\right) \\
& +\frac{\cos \left(\sqrt{\alpha_{1}} t\right)\left(18 \Omega^{8} \alpha_{2}-47 \Omega^{6} \alpha_{1} \alpha_{2}+28 F \Omega^{4} \alpha_{1} \alpha_{3}+41 \Omega^{4} \alpha_{1}^{2} \alpha_{2}-11 F \Omega^{2} \alpha_{1}^{2} \alpha_{3}-13 \Omega^{2} \alpha_{1}^{3} \alpha_{2}+F \alpha_{1}^{3} \alpha_{3}+\alpha_{1}^{4} \alpha_{2}\right) F^{2}}{\alpha_{1}\left(36 \Omega^{12}-157 \Omega^{10} \alpha_{1}+269 \Omega^{8} \alpha_{1}^{2}-226 \Omega^{6} \alpha_{1}^{3}+94 \Omega^{4} \alpha_{1}^{4}-17 \Omega^{2} \alpha_{1}^{5}+\alpha_{1}^{6}\right)} \\
& -\frac{1}{\alpha_{1}\left(4 \Omega^{2}-\alpha_{1}\right)\left(9 \Omega^{2}-\alpha_{1}\right)\left(\Omega^{2}-\alpha_{1}\right)^{4}}\left(2 7 \left(-\frac{1}{6} \alpha_{1}\left(\Omega^{2}-\alpha_{1}\right)^{2} \alpha_{2}\left(\Omega^{2}-\frac{1}{9} \alpha_{1}\right) \cos (2 \Omega t)\right.\right. \\
& \left.\left.+\left(\Omega^{2}-\frac{1}{4} \alpha_{1}\right)\left(\frac{1}{27} F \alpha_{1} \alpha_{3}\left(\Omega^{2}-\alpha_{1}\right) \cos (3 \Omega t)+\left(F \alpha_{1} \alpha_{3} \cos (\Omega t)+\frac{2}{3} \alpha_{2}\left(\Omega^{2}-\alpha_{1}\right)^{2}\right)\left(\Omega^{2}-\frac{1}{9} \alpha_{1}\right)\right)\right) F^{2}\right) \\
& +\frac{1}{12} \frac{\cos \left(\sqrt{\alpha_{1}} t\right)\left(3 A \Omega^{2} \alpha_{3}+4 \Omega^{2} \alpha_{2}-4 \alpha_{1} \alpha_{2}\right) A^{2} \Omega^{4}}{\alpha_{1}\left(\Omega^{6}-3 \Omega^{4} \alpha_{1}+3 \Omega^{2} \alpha_{1}^{2}-\alpha_{1}^{3}\right)} \\
& -\frac{9}{32 \alpha_{1}^{(3 / 2)}\left(\Omega^{2}-\alpha_{1}\right)^{3}}\left(-\frac{16}{27} \alpha_{2}\left(\sqrt{\alpha_{1}} \Omega^{2}-\alpha_{1}^{(3 / 2)}\right) \cos \left(2 \sqrt{\alpha_{1}} t\right)\right. \\
& -\frac{1}{9} \alpha_{3} A \Omega^{2} \cos \left(3 \sqrt{\alpha_{1}} t\right) \sqrt{\alpha_{1}}+\alpha_{3} A \Omega^{2} \cos \left(\sqrt{\alpha_{1}} t\right) \sqrt{\alpha_{1}}+\frac{4}{3} A \Omega^{2} t \alpha_{3} \sin \left(\sqrt{\alpha_{1}} t\right) \alpha_{1} \\
& \left.+\frac{16}{9} \alpha_{2}\left(\sqrt{\alpha_{1}} \Omega^{2}-\alpha_{1}^{(3 / 2)}\right)\right) A^{2} \Omega^{4}-\frac{1}{12} \frac{\cos \left(\sqrt{\alpha_{1}} t\right)\left(3 A \alpha_{1} \alpha_{3}-4 \Omega^{2} \alpha_{2}+4 \alpha_{1} \alpha_{2}\right) \alpha_{1} A^{2}}{\Omega^{6}-3 \Omega^{4} \alpha_{1}+3 \Omega^{2} \alpha_{1}^{2}-\alpha_{1}^{3}} \\
& +\frac{3}{8\left(\Omega^{2}-\alpha_{1}\right)^{3}}\left(\frac{4}{9} \alpha_{2}\left(\Omega^{2}-\alpha_{1}\right) \cos \left(2 \sqrt{\alpha_{1}} t\right)-\frac{1}{12} A \alpha_{1} \alpha_{3} \cos \left(3 \sqrt{\alpha_{1}} t\right)+\frac{3}{4} \alpha_{3} \cos \left(\sqrt{\alpha_{1}} t\right) A \alpha_{1}\right. \\
& \left.+A \alpha_{1}^{(3 / 2)} \sin \left(\sqrt{\alpha_{1}} t\right) t \alpha_{3}-\frac{4}{3} \alpha_{2}\left(\Omega^{2}-\alpha_{1}\right)\right) \alpha_{1} A^{2}
\end{aligned}
$$

Box I

tude on the natural frequency of system are demonstrated in Figure 8.

It is seen that the natural frequency increases by increasing the amplitude and decreases by increasing the curvature radius. The influences of the inner radius and waviness amplitude of the curved SWCNT on its natural frequency are demonstrated in Figure 9.

This figure shows that natural frequency increases by increasing the amplitude and decreases by increasing the inner radius.

To show the efficiency of the presented method for forced vibration analysis, in comparison with numerical solution, three examples are given.

\section{Example 1}

The non-dimensional transverse load, $F$, the nondimensional frequency of transverse load, $\Omega$, and the initial condition are described by $F=10$ Pico Newton, $\Omega=3$, and $A=0 \mathrm{~nm}$ respectively. By assuming Case 1 (as shown in Table 1), the equation of motion is described by:

$$
\begin{aligned}
T(t)= & -2.05 \times 10^{-4}-7.03 \times 10^{-5} t \sin (t) \\
& -8.37 \times 10^{-7} \cos ^{3}(5 t)+2.34 \\
& \times 10^{-5} \cos ^{3}(t)+5.84 \times 10^{-5} \cos ^{2}(t)+1.77 \\
& \times 10^{-6} \cos ^{2}(5 t)-4.17 \times 10^{-2} \cos (5 t)+4.18 \\
& \times 10^{-2} \cos (t) .
\end{aligned}
$$

The comparison between analytical solution and numerical solution for forced vibration analysis is presented in Figure 10. 


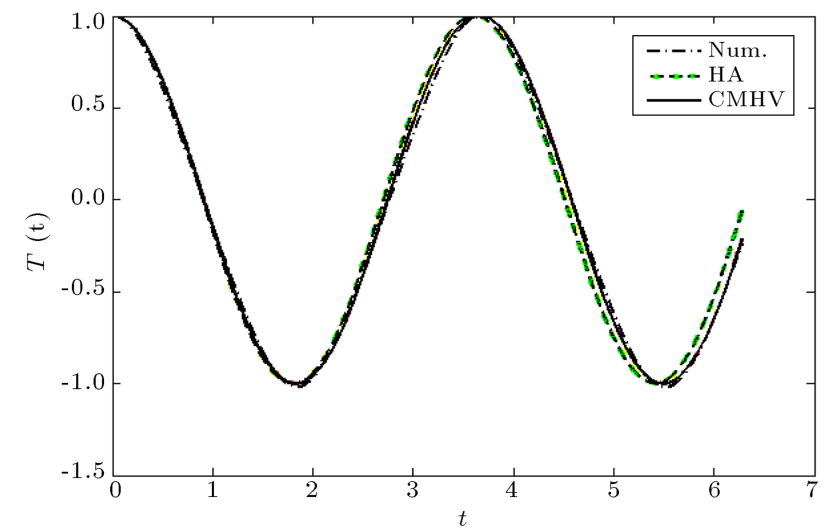

Figure 4. Comparison between HA, CMHV, and numerical solutions for free vibration analysis $(A=1 \mathrm{~nm}$, Case 1).

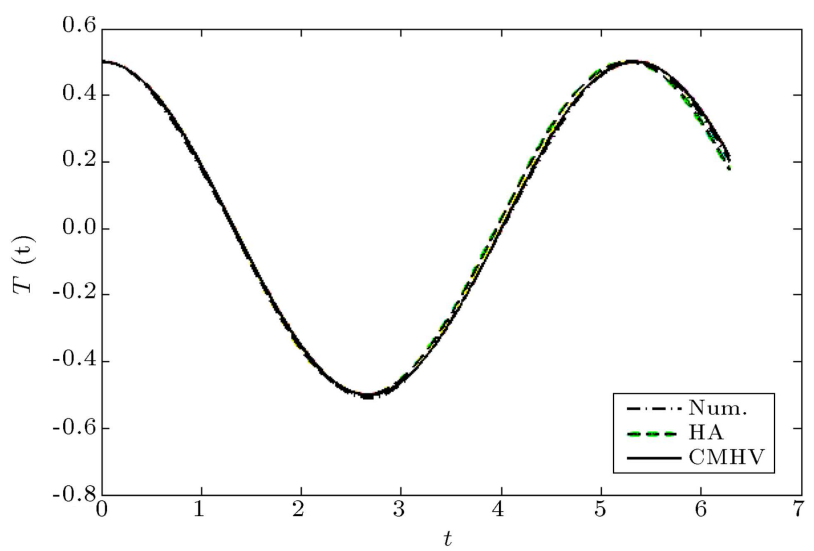

Figure 5. Comparison between HA, CMHV, and numerical solutions for free vibration analysis $(A=0.50$ nm, Case 4).

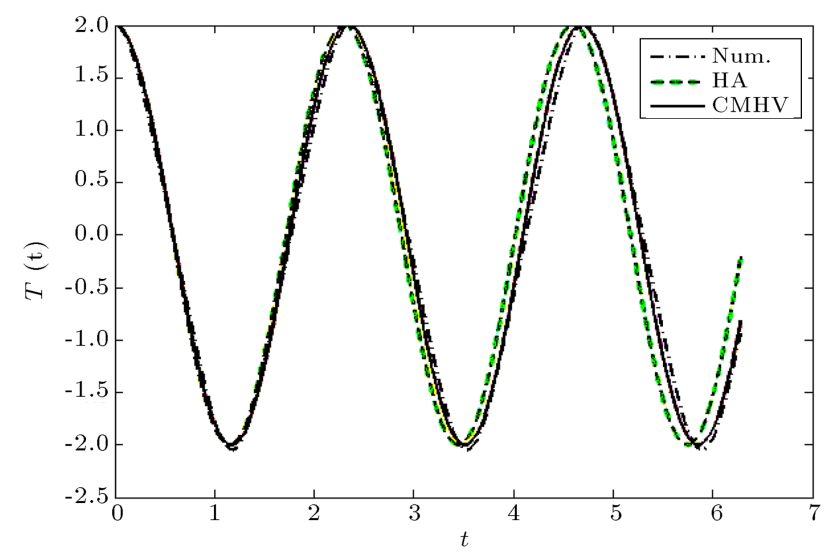

Figure 6. Comparison between HA, CMHV, and numerical solutions for free vibration analysis $(A=2 \mathrm{~nm}$, Case 9).

\section{Example 2}

The non-dimensional transverse load, $F$, the nondimensional frequency of transverse load, $\Omega$, and the initial condition in this example are described by $F=$ $1 \mathrm{pN}, \Omega=5$, and $A=0.1 \mathrm{~nm}$, respectively. By

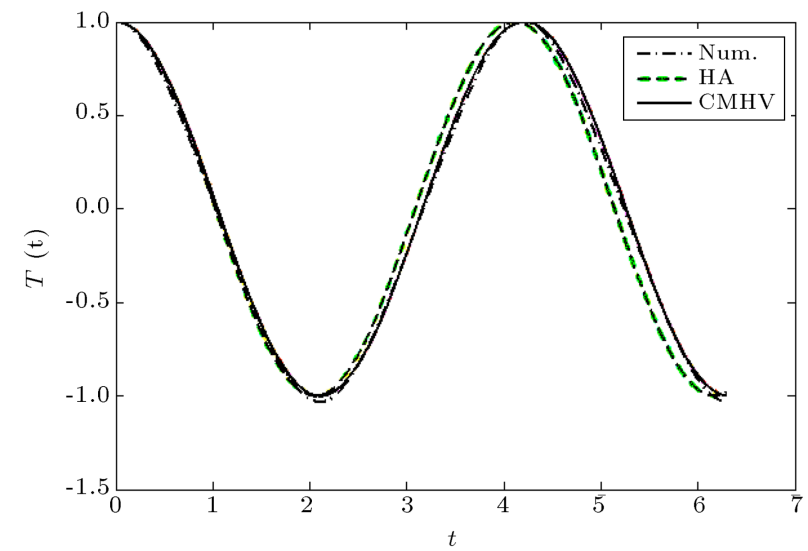

Figure 7. Comparison between HA, CMHV, and numerical solutions for free vibration analysis $(A=1 \mathrm{~nm}$, Case 10).

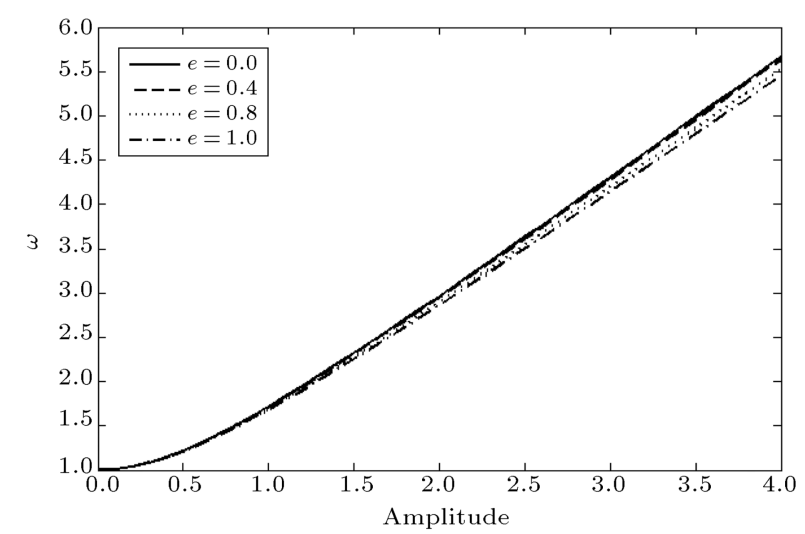

Figure 8. Influences of the curvature radius and amplitude on the natural frequency, $\omega_{\mathrm{CMHV}}\left(r_{\text {in }}=0\right)$.

assuming Case 8 (shown in Table 1 ), the equation of motion is described by:

$$
\begin{aligned}
T(t)= & -1.64 \times 10^{-3}-1.02 \times 10^{-3} t \sin (t) \\
& +3.41 \times 10^{-4} \cos ^{3}(t)+3.10 \times 10^{-6} \cos ^{2}(5 t) \\
& -7.32 \times 10^{-7} \cos ^{3}(5 t)+5.12 \times 10^{-7} \cos (2 t) \\
& -5.12 \times 10^{-9} \cos (3 t)+7.43 \times 10^{-4} \cos ^{2}(t) \\
& -4.17 \times 10^{-2} \cos (5 t)+1.42 \times 10^{-1} \cos (t) .
\end{aligned}
$$

The comparison between analytical and numerical solutions for forced vibration analysis is presented in Figure 11.

\section{Example 3}

The non-dimensional transverse load, $F$, the nondimensional frequency of transverse load, $\Omega$, and the initial condition are described by $F=1 \mathrm{pN}, \Omega=4$, and 


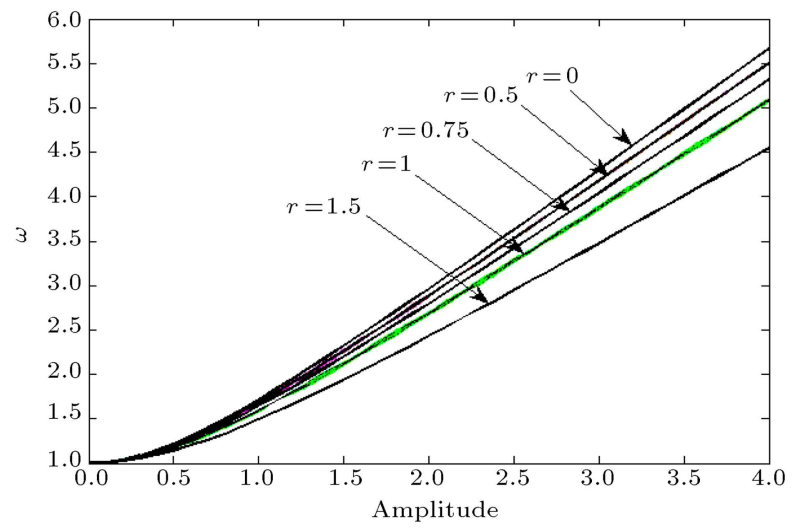

Figure 9. Influences of the inner radius and amplitude on the natural frequency, $\omega_{\mathrm{CMHV}}\left(r=r_{\mathrm{in}}\right)$.

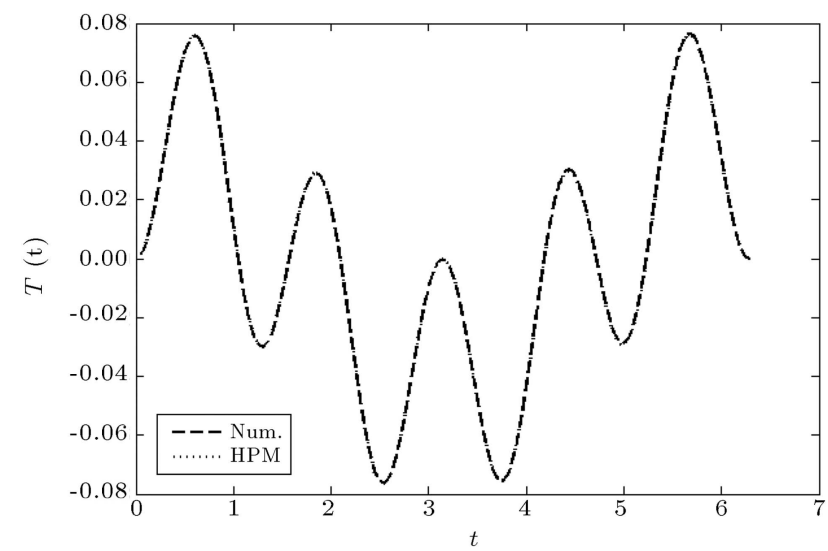

Figure 10. Time history responses of the system for Example 1.

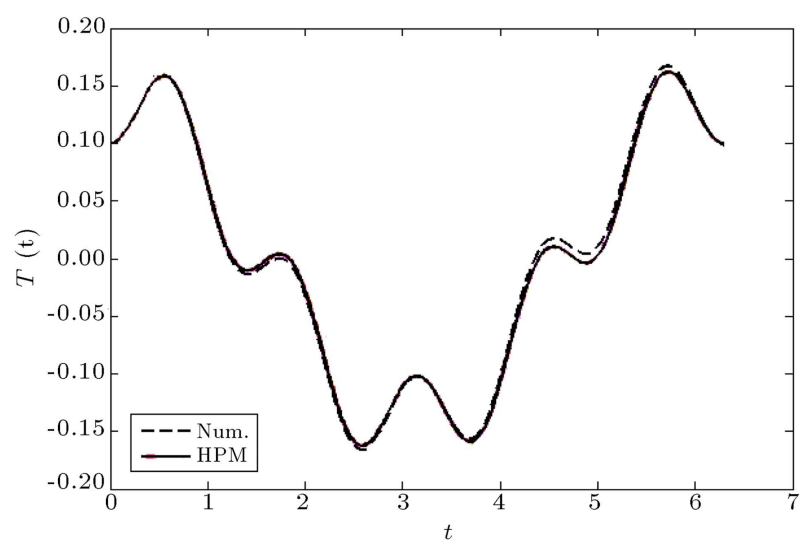

Figure 11. Time history responses of the system for Eexample 2.

$A=0.1 \mathrm{~nm}$, respectively. By assuming Case 6 (shown in Table 1), the equation of motion is described by:

$$
\begin{aligned}
T(t)= & -2.58 \times 10^{-3}-1.46 \times 10^{-3} t \sin (t)+4.88 \\
& \times 10^{-4} \cos ^{3}(t)+1.42 \times 10^{-5} \cos ^{2}(4 t)-5.36 \\
& \times 10^{-6} \cos (4 t)+1.49 \times 10^{-6} \cos (2 t)-2.39
\end{aligned}
$$

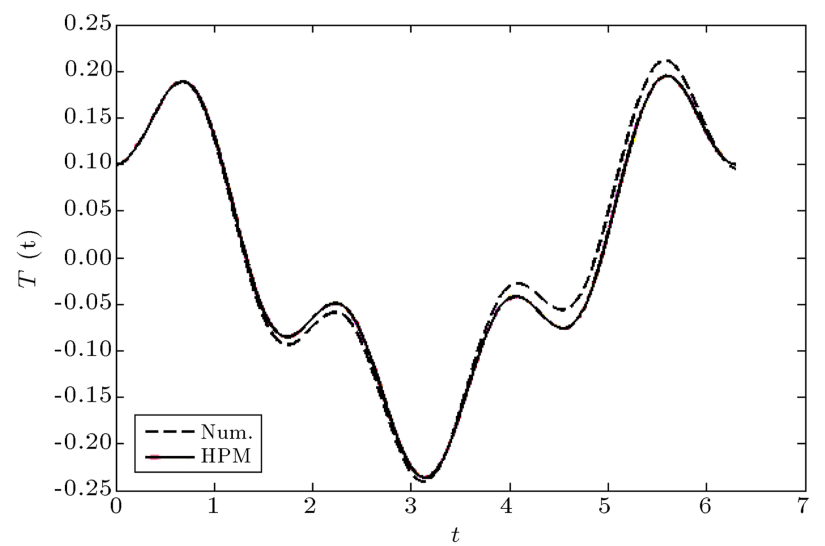

Figure 12. Time history responses of the system for Example 3.

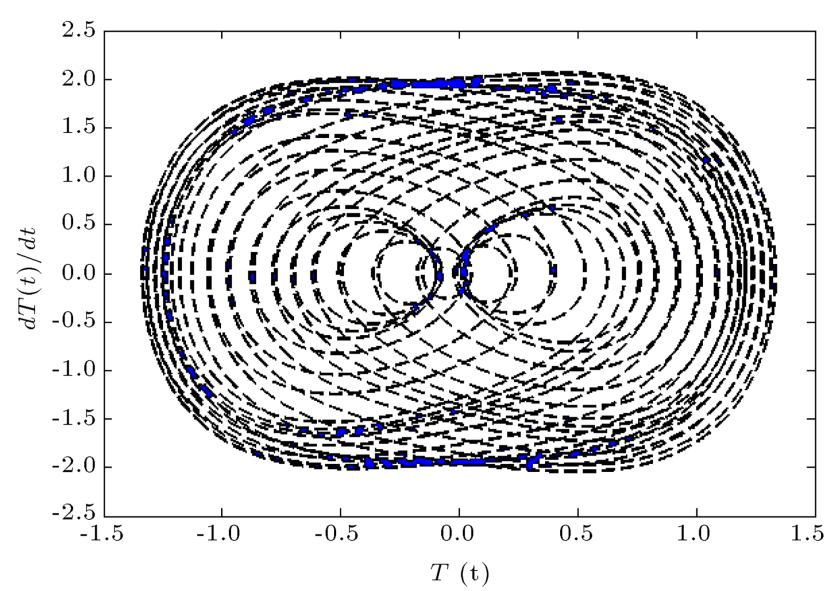

Figure 13. Phase-plane trajectory $(\Omega=1.2 \mathrm{rad} / \mathrm{s}$, $F=1 \mathrm{pN}, A=0 \mathrm{~nm}$, Case 1).

$$
\begin{aligned}
& \times 10^{-8} \cos (3 t)+1.06 \times 10^{-3} \cos _{2}(t)-6.67 \\
& \times 10^{-2} \cos (4 t)+1.68 \times 10^{-1} \cos (t) .
\end{aligned}
$$

The comparison between analytical solution and numerical solution for forced vibration analysis is presented in Figure 12.

Figures 10-12 show excellent agreement between the approximate results and those obtained by the numerical method. This agreement proves that this method is powerful and reliable in solving conservative nonlinear oscillatory systems. The phase-plane trajectories, representing the vibration performance of the system, are shown in Figures 13-15.

In Figures 13 and 14, the excitation frequency, $\Omega$, is close to the natural frequency, $\omega$, and, for this reason, we see the big amplitude in these figures. Also, in Figure 14, we have bigger excitation force, $F$, than that in Figure 13, and, as a result, we have bigger amplitude than that in Figure 13. In Figure 15, the excitation frequency, $\Omega$, is not close to the natural frequency, $\omega$, and, for this reason, we see small amplitude in the oscillation. 


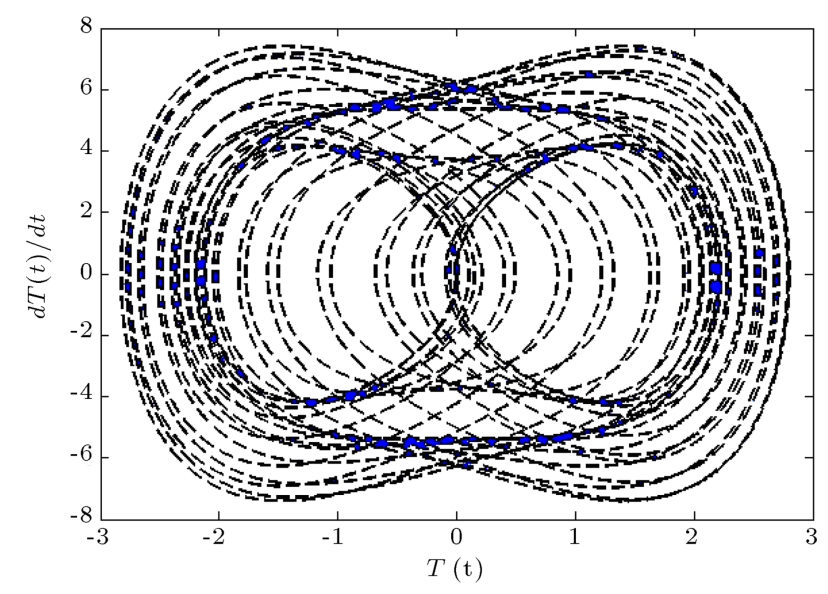

Figure 14. Phase-plane trajectory $(\Omega=1.2 \mathrm{rad} / \mathrm{s}$, $F=10 \mathrm{pN}, A=0 \mathrm{~nm}$, Case 1).

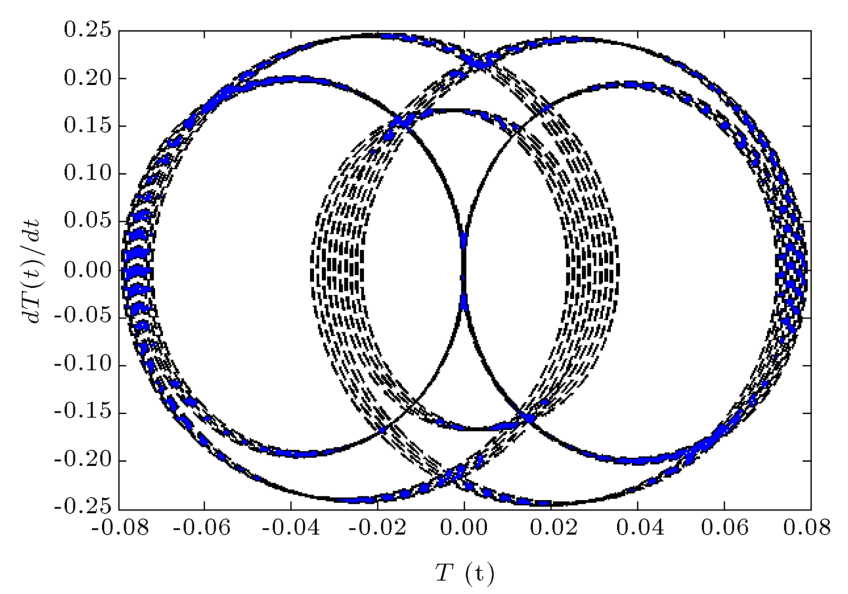

Figure 15. Phase-plane trajectory $(\Omega=5 \mathrm{rad} / \mathrm{s}$, $F=10 \mathrm{pN}, A=0 \mathrm{~nm}$, Case 1).

\section{Conclusions}

An elastic continuum approach for modeling nonlinear vibration of a single-walled carbon nanotube under harmonic excitation was presented. The carbon nanotube was modeled as a doubly clamped wavy (curved) beam. Two analytical methods were used to investigate the frequency-amplitude relationship of the system. Parametric study was performed to investigate the effects of the oscillation amplitude, curvature radius, and inner radius on the nonlinear behavior of the system. Analytical dynamic response of forced vibration was characterized by phase portrait and the time history diagrams. A numerical simulation was carried out to demonstrate the accuracy of the solutions obtained by the analytical methods.

It has been shown that the proposed approaches illustrate to be potent for the vibrational analysis of nanostructures. Finally, the present study has shown the applicability of analytical methods (HPM, HA, and $\mathrm{CMHV}$ ) in estimation of the natural frequencies and dynamic behavior of nonostructures.

\section{References}

1. Iijima, S. "Helical microtubes of graphitic carbon", Nature, 354, pp. 56-58 (1991).

2. Falvo, M.R., Clay, G.J., Taylor, R.M., Chi, V., Brooks, F.P., Washburn, S. and Superfine, R. "Bending and buckling of carbon nanotubes under large strain", Nature, 389, pp. 582-584 (1997).

3. Brischetto, S., Tornabene, F., Fantuzzi, N. and Bacciocchi, M. "Refined 2D and exact 3D shell models for the free vibration analysis of single- and double-walled carbon nanotubes" , Technologies, 3(4), pp. 259-284 (2015).

4. Brischetto, S. "A continuum shell model including van der waals interaction for free vibrations of doublewalled carbon nanotubes", Computer Modeling in Engineering and Sciences, 104(4), pp. 305-327 (2015).

5. Brischetto, S. "A continuum elastic three-dimensional model for natural frequencies of single-walled carbon nanotubes", Composites Part B: Engineering, 61, pp. 222-228 (2014).

6. Mehdipour, I., Barari, A. and Domairry, G. "Application of a cantilevered SWCNT with mass at the tip as a nanomechanical sensor", Computational Materials Science, 50, pp. 1830-1833 (2011).

7. Claeyssen, J.R., Tsukazan, T. and Coppeti, R.D. "Nonlocal effects in modal analysis of forced responses with single carbon nanotubes", Mechanical Systems and Signal Processing, 38(2), pp. 299-311 (2013).

8. Cinefra, M., Carrera, E. and Brischetto, S. "Refined shell models for the vibration analysis of multiwalled carbon nanotubes", Mechanics of Advanced Materials and Structures, 18(7), pp. 476-483 (2011).

9. Scorrano, A. and Carcaterra, A. "Semi-classical modeling of nano-mechanical transistors", Mechanical Systems and Signal Processing, 39(1-2), pp. 489-514 (2013).

10. Oveissi, S., Toghraie, D.S. and Eftekhari, A. "Longitudinal vibration and stability analysis of carbon nanotubes conveying viscous fluid", Physica E: Lowdimensional Systems and Nanostructures, 38, pp. 275283 (2016).

11. Setoodeh, A.R. and Shojaee, M. "Application of TWDQ method to nonlinear free vibration analysis of FG carbon nanotube-reinforced composite quadrilateral plates", Thin-Walled Structures, 108, pp. 1-11 (2016).

12. Fu, Y.M., Zhou, H.Q. and Zhang, P. "Nonlinear free vibration of nanowires including size effects", Micro \& Nano Letters, 17, pp. 348-352 (2012).

13. Wang, B., Deng, Z.C. and Zhang, K. "Nonlinear vibration of embedded single-walled carbon nanotube with geometrical imperfection under harmonic load based on nonlocal Timoshenko beam theory", Applied Mathematics and Mechanics, 34, pp. 269-280 (2013).

14. Shahba, A., Attarnejad, R., Tavanie Marvi, M. and Shahriari, V. "Free vibration analysis of non-uniform thin curved arches and Rings using Adomian modified 
decomposition method", Arabian Journal of Science and Engineering, 35, pp. 965-976 (2012).

15. Mayoof, F.N. and Hawwa, M.A. "Chaotic behavior of a curved carbon nanotube under harmonic excitation", Chaos, Solitons and Fractals, 42, pp. 1860-1867 (2009).

16. Joshi, A.Y., Bhatnagar, A., Sharma, S.C. and Harsha, S.P. "Vibratory analysis of a doubly clamped wavy single walled carbon nanotube based nano mechanical sensors", International Journal of Engineering Science and Technology, 2(5), pp. 993-1000 (2010).

17. Mehdipour, I., Barari, A., Kimiaeifar, A. and Domairry, G. "Vibrational analysis of curved singlewalled carbon nanotube on a Pasternak elastic foundation", Advances in Engineering Software, 48, pp. 1-5 (2012).

18. Cveticanin, L., Kalami Yazdi, M. and Askari, H. "Analytical approximations to the solutions for a generalized oscillator with strong nonlinear terms", Journal of Engineering Mathematics, 77(1), pp. 211223 (2012).

19. Yildirim, A., Askari, H., KalamiYazdi, M. and Khan, Y. "A relationship between three analytical approaches to nonlinear problems", Applied Mathematics Letters, 25(11) pp. 1729-1733 (2012).

20. Navarro, H.A. and Cveticanin, L. "Amplitudefrequency relationship obtained using Hamiltonian approach for oscillators with sum of non-integer order nonlinearities", Applied Mathematics and Computation, 291, pp. 162-171 (2016).

21. Akbarzade, M. and Kargar, A. "Accurate analytical solutions to nonlinear oscillators by means of the Hamiltonian approach", Mathematical Methods in the Applied Sciences, 34(17), pp. 2089-2094 (2011).

22. Mehdipour, I., Ganji, D.D. and Mozaffari, M. "Application of the energy balance method to nonlinear vibrating equations", Current Applied Physics, 10, pp. 104-112 (2010).

23. Ganji, S.S., Barari, A. and Ganji, D.D. "Approximate analysis of two mass-spring systems and buckling of a column", Computers and Mathematics with Applications, 61, pp. 1088-1095 (2011).

24. Simsek, M. "Nonlinear free vibration of a functionally graded nanobeam using nonlocal strain gradient theory and a novel Hamiltonian approach", International Journal of Engineering Science, 105, pp. 12-27 (2016).

25. He, J.H. "Hamiltonian approach to nonlinear oscillators", Physics Letters A, 374, pp. 2312-2314 (2010).

26. He, J.H. "Homotopy perturbation technique", Computer Methods in Applied Mechanics and Engineering, 178, pp. 257-262 (1999).

27. He, J.H. "A coupling method of homotopy technique and perturbation technique for nonlinear problems", International Journal of Non-Linear Mechanics, 35(1), pp. 115-123 (2000).

28. Askari, A.R. and Tahani, M. "Stability analysis of electrostatically actuated nano/micro-beams under the effect of van der Waals force, a semi-analytical approach", Communications in Nonlinear Science and Numerical Simulation, 34, pp. 130-141 (2016).

29. Sheikholeslami, M., Ashorynejad, H.R., Ganji, D.D. and Yildirim, A. "Homotopy perturbation method for three-dimensional problem of condensation film on inclined rotating disk", Scientia Iranica A, 19(3), pp. 437-442 (2012).

30. Khan, Y., Vázquez-Leal, H. and Faraz, N. "An efficient new iterative method for oscillator differential equation", Scientia Iranica A, 19(6), pp. 1473-1477 (2012).

31. Baghani, M., Asgarshamsi, A. and Goharkhah, M. "Analytical solution for large amplitude vibrations of microbeams actuated by an electro-static force", Scientia Iranica B, 20(5), pp. 1499-1507 (2013).

32. Daeichi, M. and Ahmadian, M.T. "Application of variational iteration method to large vibration analysis of slenderness beams considering mid-plane stretching", Scientia Iranica B, 22(5), pp. 1911-1917 (2015).

33. Ganji, S.S., Barari, A. and Ganji, D.D. "Approximate analysis of two mass-spring systems and buckling of a column", Computers and Mathematics with Applications, 61, pp. 1088-1095 (2011).

34. Fereidoon, A., Rostamiyan, Y., Akbarzade, M. and Ganji, D.D. "Application of He's homotopy perturbation method to nonlinear shock damper dynamics", Archive of Applied Mechanics, 80(6), pp. 641-649 (2010).

35. Akbarzade, M. and Khan, Y. "Dynamic model of large amplitude non-linear oscillations arising in the structural engineering: Analytical solutions", Mathematical and Computer Modelling, 55, pp. 480-489 (2012).

36. Khan, Y., Akbarzade, M. and Kargar, A. "Coupling of homotopy and the variational approach for a conservative oscillator with strong odd-nonlinearity", Scientia Iranica A, 19(3), pp. 417-422 (2012).

37. Akbarzade, M. and Langari, J. "Determination of natural frequencies by coupled method of homotopy perturbation and variational method for strongly nonlinear oscillators", Journal of Mathematical Physics, 52(2), 023518 (2011).

\section{Biographies}

Mehdi Akbarzade obtained his MS degree in Mechanical Engineering from Mazandaran University, Iran. He is currently PhD student in the Department of Mechanical Engineering, Ferdowsi University of Mashhad, Iran. His main field of research is focused on nonlinear dynamics and vibration. He has published more than 25 papers in this subject area.

Anooshiravan Farshidianfar is Full Professor in the Department of Mechanical Engineering, Ferdowsi University of Mashhad, Iran. He received his BSc and 
MSc degrees in Mechanical Engineering from Tehran University, Iran. He continued his graduate studies abroad and graduated with a PhD degree in Mechanical Engineering from University of Bradford, UK, 2001.

Dr. Farshidianfar's research interests include vibration analysis and fault diagnosis, control of vibration, engineering acoustics and noise control, nonlinear vibration and chaos, rotor dynamics, condition monitoring, experimental modal analysis, nanomechanics and design, and manufacturing of plate heat exchanger.
Masoud Tahani is Full Professor in the Department of Mechanical Engineering, Ferdowsi University of Mashhad, Iran. He received his $\mathrm{MSc}$ and $\mathrm{PhD}$ degrees in Mechanical Engineering from Sharif University of Technology, Iran. Also, he is Head of the Department of Mechanical Engineering at Ferdowsi University of Mashhad, Iran. His research interests include mechanics of composite materials, smart materials, elasticity (plates and shells), dynamics and vibration, nanomechanics, and biomechanics. 